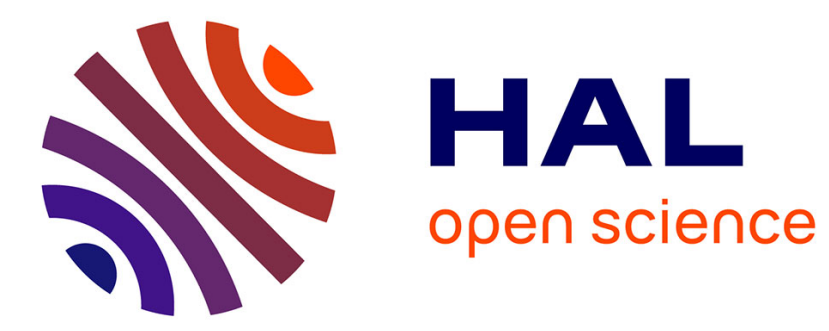

\title{
Spectral properties of dynamical power systems
}

Felix Koeth, Nicolas Retière

\section{- To cite this version:}

Felix Koeth, Nicolas Retière. Spectral properties of dynamical power systems. 13th IEEE PowerTech, Jun 2019, Milan, Italy. hal-02179204

\section{HAL Id: hal-02179204 \\ https://hal.science/hal-02179204}

Submitted on 17 Jul 2019

HAL is a multi-disciplinary open access archive for the deposit and dissemination of scientific research documents, whether they are published or not. The documents may come from teaching and research institutions in France or abroad, or from public or private research centers.
L'archive ouverte pluridisciplinaire HAL, est destinée au dépôt et à la diffusion de documents scientifiques de niveau recherche, publiés ou non, émanant des établissements d'enseignement et de recherche français ou étrangers, des laboratoires publics ou privés. 


\section{Spectral properties of dynamical power systems}

\author{
Felix Koeth \\ Univ. Grenoble Alpes, CNRS, Grenoble INP*, \\ G2Elab, F-38000 Grenoble, France \\ Email: felix.koeth@g2elab.grenoble-inp.fr
}

\author{
Nicolas Retiere \\ Univ. Grenoble Alpes, CNRS, Grenoble INP*, \\ G2Elab, F-38000 Grenoble, France \\ Email: nicolas.retiere@univ-grenoble-alpes.fr
}

\begin{abstract}
The integration of new renewable energies sources in the power system will stress the power system even more. To assure save operation, the stability of the power system has to be studied in detail. A well known feature in the power system are the inter- and intra-area oscillations of generators. This work investigates the origin and properties of this oscillations from a fundamental point of view, applied to a realistic power system test case.
\end{abstract}

\section{INTRODUCTION}

The power system is probably the worlds most complicated human made system and of vital importance for modern society. Stable operation of this system is required to ensure safety and well being a large group of people. But this system faces challenges due to climate change, as the default means of producing energy have to change to renewable energies with lower carbon footprints. This changes further complicate the problems of stable operation. So, more than ever, the stability of the power system has to be investigated and understood. The power system consist of a large number of different, coupled devices. The many different scales and complicated, time dependent dynamics result in a very complex system. To assess the interplay between the dissimilarity and the coupling of the system, simplified power system models are often used. The stability of this systems is usually investigated by finding a synchronized solution [1] and investigating the transient stability of this solutions after large perturbations [2], [3], [4]. Also, the small signal stability from small perturbations around the operating point has to be taken into account [5].

An interesting property found in stability investigations are the power system oscillations [6]. The fast intra-area oscillations describe the dynamics of neighboring generators, which swing against each other. The intra-area oscillators are slower and deal with large scale interactions of coherent regions of generators. For large-scale control of the power system, these clusters are often bundled to a aggregated generator and the intra-area modes describe the oscillations between bundled oscillators. Understanding the formation and properties of these clusters and the coherent dynamics are thus vital for the control and design of the power system. The objective of this work is to investigate how the dynamical parameters and topology of a generator network influences the spectral properties and the eigenvectors, which are the basis for the formation of coherent clusters.

\footnotetext{
*Institute of Engineering Univ. Grenoble Alpes
}

In this work, the spectral properties of a specific IEEE test case are investigated. At first, modeling background and theoretical results are introduced. Then, numerical experiments on the test case are carried out. The main question is the influence on the spectral properties of the parameters from the system, mainly the generator properties and the topology of the network. Also, the robustness of the spectrum under parameter changes is investigated. In a last step, the existence of coherency in the eigenvectors is investigated.

\section{Modeling AND THEORY}

In this section, the simplified power system model is introduced. The chosen model is the structure preserving model (SP-Model). The model is linearized and the mathematical problem statement is explained. Also, a review of the known analytical properties of the mathematical problem is given.

\section{A. SP Model and quadratic eigenvalue problem}

A common way to investigate fundamental properties of power systems and the interplay between the topology, parameters and the system dynamics is to use simplified power system models. Many aspects of complex power system models, as the reactive power flow, load dynamics and active control effects are ignored. The differential equations describing the dynamics of the phases $\theta_{i}$ at the generator $\left(\mathcal{V}_{G}\right)$ and load $\left(\mathcal{V}_{L}\right)$ nodes $i$ are given by [7], [8], [9]:

$$
\begin{aligned}
M_{i} \ddot{\theta}_{i}+D_{i} \dot{\theta}_{i}=\omega_{i}-\sum_{j} a_{i j} \sin \left(\theta_{i}-\theta_{j}\right) & i \in \mathcal{V}_{1} \\
D_{i} \dot{\theta}_{i}=\omega_{i}-\sum_{j} a_{i j} \sin \left(\theta_{i}-\theta_{j}\right) & i \in \mathcal{V}_{2}
\end{aligned}
$$

With the inertia $M$, the damping $D$, the power input/output $\omega$ and the adjacency matrix $A$ with entries $a_{i j}$ of the weighted graph $\mathcal{G}$, which is given in power system nomenclature as $a_{i j}=U_{i} U_{j} \operatorname{Im} Y_{i j}$ with the bus Voltages $U$ and the nodal admittance matrix of the network $Y$. For the remainder of this work, the damping of the load nodes is assumed to be zero. The resulting equations for the load nodes are thus purely algebraic. The SP-Model was extensively used in the studies linked in the introduction, especially for transient stability studies. It is said to capture the dynamics of the first swing of generators reasonably well. Usually, no external control is used. The damping term $D \dot{\theta}$ incorporates not only mechanical 
damping, but also the rotor-stator flux effects and machine specific controls.

For small perturbations around a fixed point, the small angle approximations leads to the following linear system:

$$
M \ddot{\theta}+D \dot{\theta}+L \theta=\omega
$$

With $L$ being the Laplacian matrix of the Graph $G$. Depending on the model, the diagonal matrices $M$ and $D$ have have zero entries for the nodes $i \in \mathcal{V}_{G}$. The main interest is the dynamical behavior of the generators, so the responsefree system with $\omega=0$ is considered. The solutions to this equation can be found by solving the following quadratic eigenvalue problem (QEP):

$$
\left(\lambda^{2} M+\lambda D+L\right) x=0
$$

In accordance to the eigenvalue problem, we call $\lambda_{k}$ the eigenvalues and $x_{k}$ the (right) eigenvector of the QEP. Due to the quadratic nature of the problem, we have $2 n$ solutions to the quadratic eigenvalue problem. Using $\gamma=\left[\gamma_{1} \ldots \gamma_{2 n}\right]$ as a vector of arbitrary constants related to the initial values of the problem, the solution $\theta$ can then be calculated as [10]:

$$
\theta(t)=\sum_{k}^{2 n} \gamma_{k} x_{k} \exp \left(\lambda_{k} t\right)
$$

From equation (5), coherency in the phases of the power system can be defined. If, for a given mode $\lambda_{k}$, some components of the eigenvector $x_{k}$ are (roughly) identical, this modes will show similar response to a excitation of this mode. As the eigenvectors of the quadratic eigenvalue problem are generally complex, coherent behavior can be observed if the complex phase and magnitude of eigenvector components are similar. The quadratic (and higher order) eigenvalue problems are usually calculated by using the companion form, where the QEP with $n \times n$ matrices is transformed to a generalized eigenvalue problem with $2 n \times 2 n$ matrices [11].

\section{B. Kron reduction}

As previously mentioned, the assumption of undamped loads results in algebraic equations for the load nodes. This load nodes will thus not exhibit a dynamic response, but change infinity fast. This can be used to simplify the systems equations, by writing the Laplacian matrix $L$ and the inertia $M$ in block form and solving the eigenvalue problem for the $M, L$ matrix pencil ${ }^{1}$ :

$$
\left(\begin{array}{cc}
L_{G} & L_{G L} \\
L_{G L}^{T} & L_{L}
\end{array}\right)\left(\begin{array}{l}
v_{G} \\
v_{L}
\end{array}\right)=\lambda^{2}\left(\begin{array}{cc}
M_{G} & 0 \\
0 & 0
\end{array}\right)\left(\begin{array}{l}
v_{G} \\
v_{L}
\end{array}\right)
$$

The eigenvector for the load nodes can be removed by inserting the second row of the matrix equation in the first row, resulting in:

\footnotetext{
${ }^{1}$ For simplicity, the damping is ignored here, but the calculations are identical for damped generators.
}

$$
L_{G} v_{G}+L_{G L} v_{L}=\underbrace{L_{G} v_{G}-L_{G L} L_{L}^{-1} L_{G L}^{T} v_{G}}_{=L_{\mathrm{red}} v_{G}}=\lambda^{2} M_{G} v_{G}
$$

Which is identical to the Shur complement of the Laplacian matrix with respect to the sub-matrix of load nodes. The reduced Laplacian $L_{\text {red }}$ is of size $n_{G} \times n_{G}$. The reduced matrix is still a Laplacian matrix [12].

This process is identical to the well known Kron reduction, where all load nodes are removed and the admittances between the generators are augmented. This process results in a complete graph. The underlying topological properties are encoded in the changed edge-weights of the complete graph. The other matrices of the QEP are now the submatrices $M_{G}$ and $D_{G}$, which are full diagonal sub matrices of the generator machine properties.

\section{Theoretical background}

The main known properties of the quadratic eigenvalue problem are discussed in [10]. For the reduced eigenvalue problem stated here, the following properties of the QEP are respected:

1) $M_{G}$ is non-singular - $2 n_{G}$ finite eigenvalues

2) $M_{G}, D_{G}, L_{\text {red }}$ are real - eigenvalues are real or complex conjugate pairs

3) $M_{G}$ hermitian and positive definite, $D_{G}$ and $L_{\text {red }}$ positive semi-definite - The real parts of the eigenvalues are non-positive

A fourth condition is known as the overdamped condition, named after the related phenomenon in mechanical systems. For this condition, $M_{G}$ and $D_{G}$ have to be symmetric and positive definite and $L_{\text {red }}$ is positive semi-definite. Additionally, $\gamma(M, D, L)>0$, with $\gamma$ given as:

$$
\gamma(M, D, L)=\min _{\|x\|_{2}=1}\left(\left(x^{*} D x\right)^{2}-4\left(x^{*} M x\right)\left(x^{*} L x\right)\right)
$$

This requirement can be studied by a simple approximation to the optimization problem. An upper $\gamma \geq \gamma_{\text {up }}$ bond for the condition can be verified to be:

$$
\gamma_{\text {up }}(M, D, L)=\lambda_{D, \min }^{2}-4 \lambda_{M, \max } \lambda_{L, \max }>0
$$

Here, $\lambda_{X, \min / \max }$ is the smallest/largest eigenvalue of the matrix $X$. Overdamped behavior can be archived by increasing the damping coefficient of the generators or by lowering the inertia or connectivity of the network.

\section{NUMERICAL RESULTS}

\section{A. The IEEE 145 system}

The objective of this work is to investigate how the parameters of the network and the generators influence the spectral and coherent properties of the quadratic eigenvalue problem. For this, a suitable test case and meaningful parameters have to be found. While the nodal admittance matrix $Y$, the power in- and outputs $\omega$ and the voltages $U_{i}$ are known or can be 


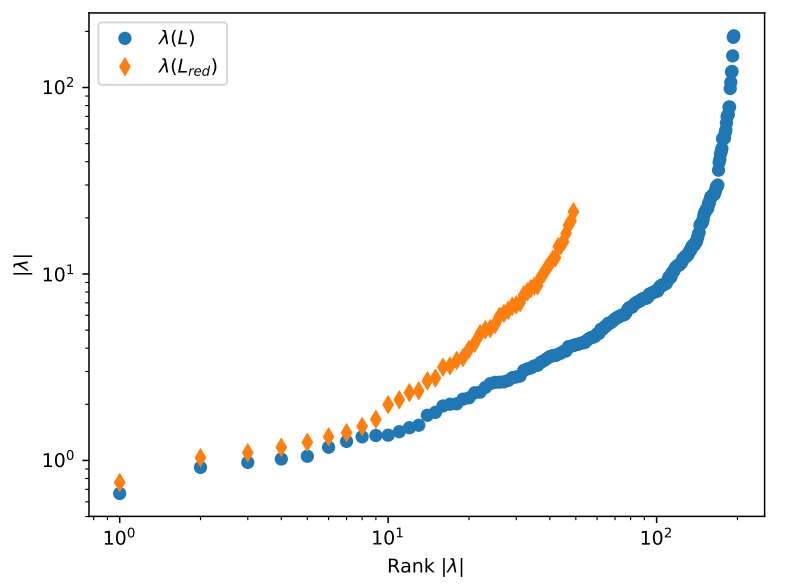

Fig. 1. Spectrum of Laplacian Matrix $L$ and reduced matrix $L_{\text {red }}$.

calculated by the default load-flow equations, the dynamic parameters are not always well known. In this study, the methods to calculate parameters found in [9], [5] are used. The main test system is dynamic 50 generator test case (sometimes called IEEE 145 system) [13]. It features 50 generators and has 145 buses. The system is mainly used for dynamical studies and some generator data is known in literature.

\section{B. The Laplacian spectrum}

In figure 1, the spectrum $\lambda(L)$ of the Laplacian matrix $L$ is shown, ranked by the absolute value. Not included is the zero eigenvalue of the Laplacian matrix. It can be observed that the first few eigenvalues are relatively small and increase slowly, which is typical for Laplacian matrices of power systems.

Also included in Graph 1 is the spectrum of the Kron reduced Laplacian $L_{\text {red }}$, as described in the theory. The resulting reduced system is less complex (as it has less nodes). The full system has a larger maximum ranked eigenvalue, but for small ranked eigenvalues, the reduced spectrum is larger, corresponding to a higher connectivity of the network.

\section{Inertia}

At first, the influence of the inertia in the spectrum is investigated. For that, the damping matrix is set to zero at first. To assess how the parameter of the inertia (e.g. the dissimilarities of the diagonal entries of $M$ ) influence the spectrum, the inertia $M$ is perturbed to $M^{\prime}$ by $\alpha=\left(\alpha_{1}, \ldots, \alpha_{N_{G}}\right)$, with $M^{\prime}=\operatorname{diag}\left(M_{1} \alpha_{1}, \ldots, M_{N_{G}} \alpha_{N_{G}}\right)$ and uniform distributed $\alpha$ with $\alpha \in[0.5,2.0]$. Figure 2 shows the original spectrum and spectra of 200 realizations with different $M_{i}$. It can be noted that for smaller ranks, the differences in spectra are relatively small. At the same time, the range of eigenvalues grows larger for higher ranked eigenvalues.

The highest ranked eigenvalues are linked to intra-area oscillations, where individual generators oscillate against each other. This localized dynamics is stronger influenced by the inertia, which is a local effect on a single node.

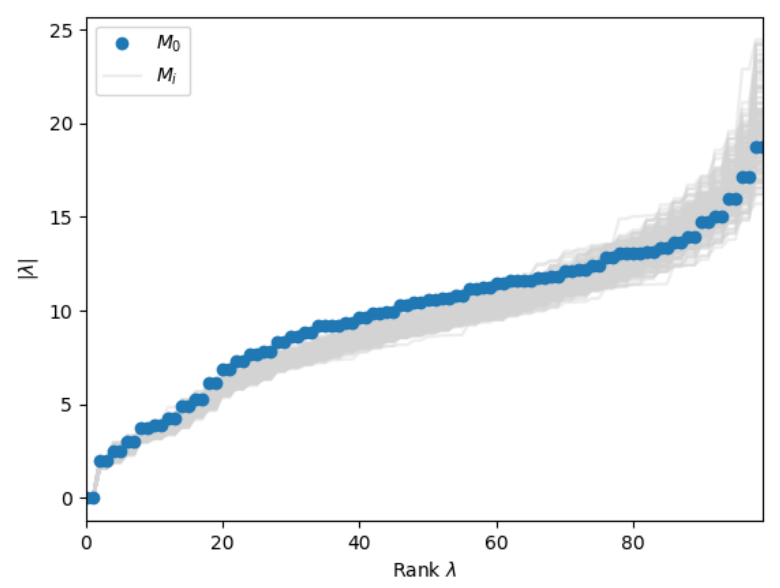

Fig. 2. Spectrum of eigenvalues of matrix pair $(M, L)$ for IEEE 145 system with randomly perturbed inertia $M$. The grey lines indicate perturbed spectra.

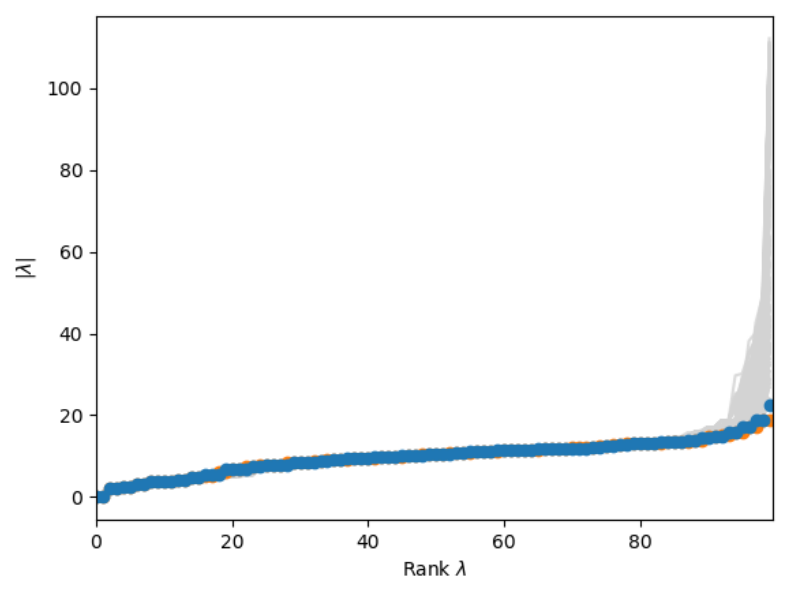

Fig. 3. Spectrum of eigenvalues of matrix pencil $(M, D, L)$ for IEEE 145 system with randomly perturbed damping $D$. The grey lines indicate perturbed spectra.

\section{Damping}

One major simplification up to this point is the fact that the generators were undamped. Realistic generators are damped, to reduce the instabilities caused by perturbations from their operating point. Here, the interest is how this intrinsic damping influences the spectra of power systems.

Similarly to the previous section, figure 3 shows the spectra for different damping realizations around the original damping ratio $D_{0}$. Additionally, spectra without any damping is included. Here, the distribution of $\alpha$ is chosen larger as $\alpha \in[0.25,4.0]$. Two observations are directly clear: even though the random range is larger, the difference seems, for the most part, to be smaller for different damping perturbations. Also, the difference in the spectrum without damping and the default damping parameter is very small. As before, 


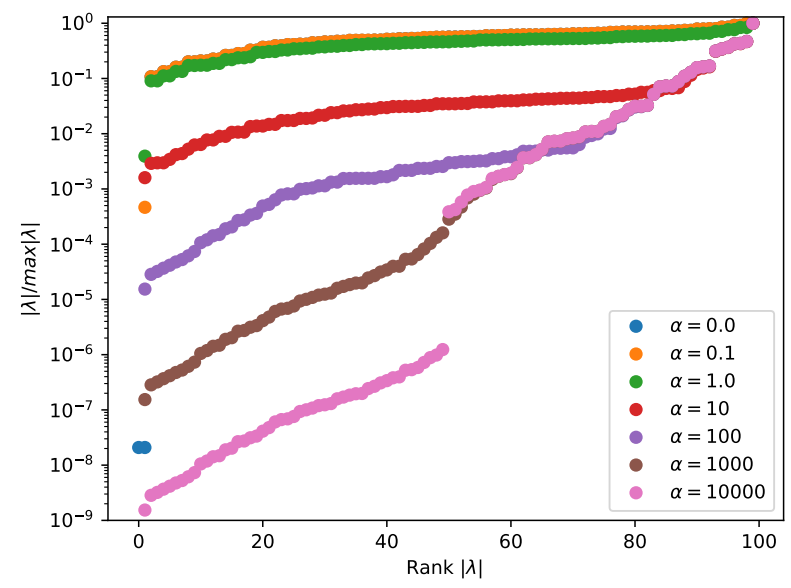

Fig. 4. Normalized spectra for different damping factors $\alpha$.

one can notice the difference for larger and smaller damped eigenvalues. Larger damped eigenvalues seem more affected by the localized parameters of $D$, while the lower ranked eigenvalues are almost unchanged and more depended on the structure of the underlying network.

The observations indicate that the spectrum is not at all overdamped. From equation (7), one can estimate that overdamped behavior would be reached if the eigenvector of $D$ is multiplied by a factor of $\alpha_{O D} \approx 1130$. This is very far from the assumed conditions in the power system and one can assume that power systems are not overdamped. The spectra for under- and overdamped behavior is shown in figure 4, where the damping matrix $D$ is calculated by $D_{0} \alpha$, with different values for $\alpha$. Again, it is clear that low to moderate values of $\alpha$ does not change the spectrum significantly. For very large $\alpha$, the observations from the theory are observable. The eigenvalues turn purely real and the gap between the first $n=50$ and last $n$ eigenvalues is clearly present.

\section{E. Sensitivity}

The previous sections show that the introduction of inertia and damping changes the results of the quadratic eigenvalue problem, but the general shape of the spectra seems to be dominated by the topology of the network. To answer which parameter, damping or inertia, influences the system stronger, the following experiment was carried out:

1) The spectra $\lambda_{0}\left(M_{0}, D_{0}, L_{r e d}\right)$ was calculated.

2) The diagonal matrices $M_{0}$ and $D_{0}$ were perturbed by random, uniform distributed vectors $r_{M}$ and $r_{D}$ to $M_{i}=$ $M_{0} r_{M, i}$ and $D_{i}=D_{0} r_{D, i}$.

3) The spectra $\lambda_{i}^{M}\left(M_{i}, D_{0}, L_{\text {red }}\right), \lambda_{i}^{D}\left(M_{0}, D_{i}, L_{\text {red }}\right)$ and $\lambda_{i}^{M, D}\left(M_{i}, D_{i}, L_{\text {red }}\right)$ were calculated for 200 different realisations.

4) The average differences from $\left(\lambda_{i}-\lambda_{0}\right)$ and variances were calculated for all cases of $\lambda_{i}$.

The resulting graphs are shown in figure 5. All perturbations $r_{i}$ are uniform distributed $0.5<r_{i}<1.5$. From the figure,
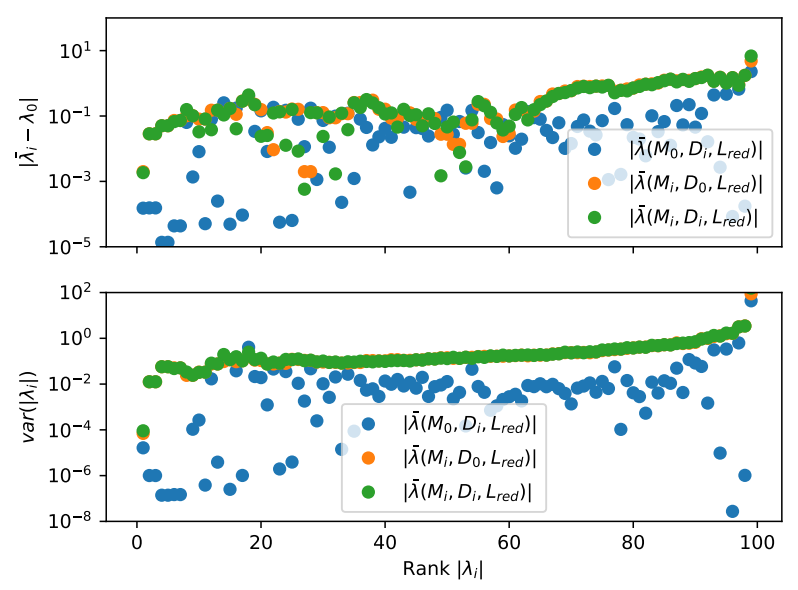

Fig. 5. Average spectral difference and variance of perturbed spectra for perturbed $M, D$ or both.

it appears that changing both $M$ and $D$ has a very similar effect to only changing the inertia. Only changing the $D$ has a much smaller effect, as the variance and the difference to the unperturbed values is much lower. While the behavior of the individual ranks are not directly clear, especially the highest ranked eigenvalue has the largest difference and variance, for all considered eigenvalues.

Another tool to investigate the global sensitivity of matrices, and in more general matrix pencils, are the pseudospectra [14], which gives insights on the region in which eigenvalues change under matrix perturbations with a certain strength. For the power system used here, the pseudo-spectrum is given in figure 6. In this figure, the markers show the real- and imaginary-part of the quadratic eigenvalue problem. The colors correspond to a perturbation strength of the matrices $M, D$ and $L$ and the perturbed eigenvalues will lie in a region corresponding to the color.

From the pseudo-spectrum, it is clear that the eigenvalues with a larger negative real part can be perturbed with weaker perturbations. At the same time, changing this value will not fundamentally alter the dynamics of the full system. The more interesting eigenvalues are those with a small real part. To perturb this eigenvalues, much larger perturbations are necessary.

From this observations, the spectral properties seem to depend mostly on the structure of the underlying network, with the exception of the highest ranked eigenvalues. In general, the inertia seems to have a bigger effect on the spectrum then the damping and for small damping values, the damping can be neglected. The next question is how do the parameters influence the coherency, or the eigenvector structure of the system.

\section{COHERENCY OBSERVATIONS}

The general structure of the eigenvalues for all modes of the given problem is shown in figure 7 . The color indicates 


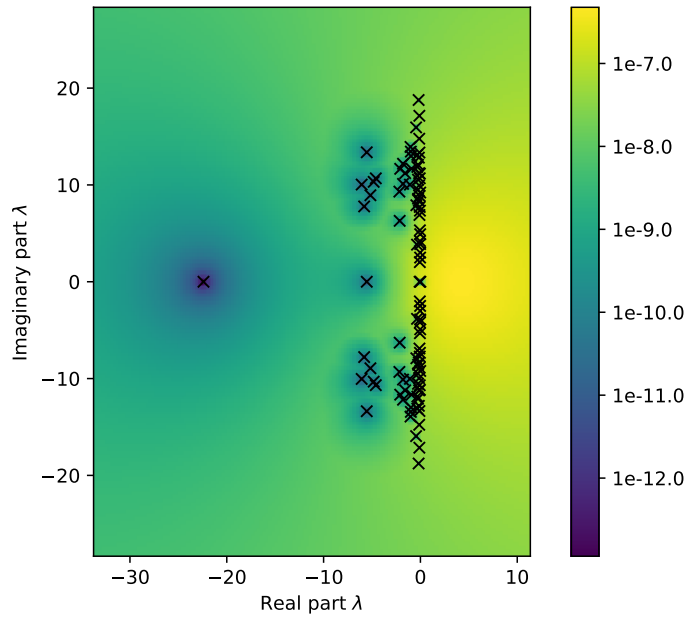

Fig. 6. Pseudo-spectrum of quadratic eigenvalue problem. Marker indicate the original eigenvalues, the color indicate the region in which the eigenvalue can fall after a perturbation of strength corresponding to the color.

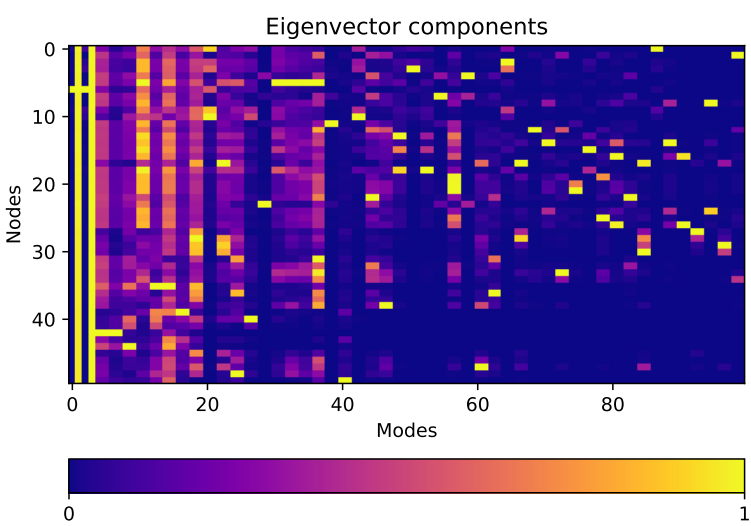

Fig. 7. Magnitude of eigenvector components for all modes in the IEEE 145 power system.

the magnitude of the eigenvector components for each mode. For smaller modes, most nodes are active. Similar values can be found for some bands in the first modes. At higher modes $(>60)$, the dynamics are more isolated. Only a few nodes oscillate at that mode at all.

On the graph, the phases and magnitudes of the eigenvector components of the second smallest eigenvalue (called Fielder vector) are shown in figure 8 , where the color of the nodes corresponds to the complex phase while the size of the nodes is the complex magnitudes. To improve observability, only edge-nodes with a edge weight ${ }^{2}$ over 0.5 are shown in this figure. The isolated nodes without any connections are only weakly connected to the rest of the system. Nodes with a

\footnotetext{
${ }^{2}$ The edge weight between two nodes is proportional to the inverse of the admittance between the two nodes.
}

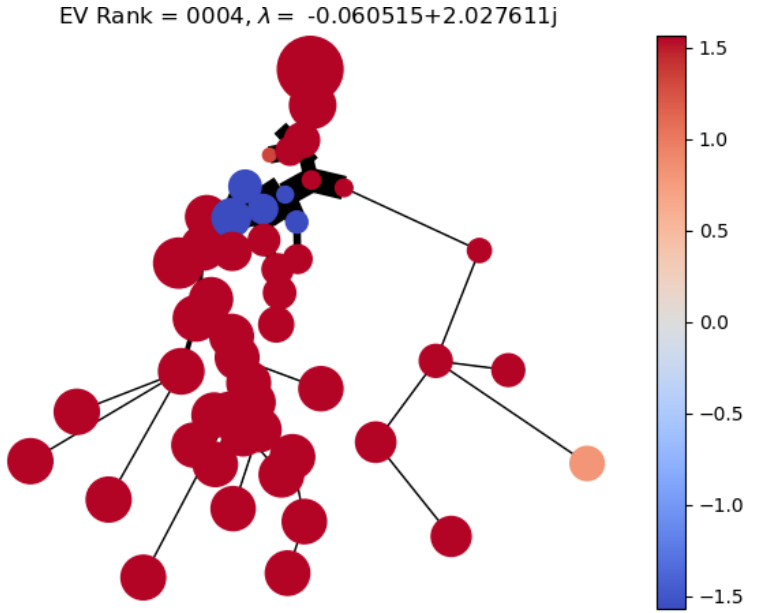

Fig. 8. Phase and magnitude of fourth ranked eigenvalue.

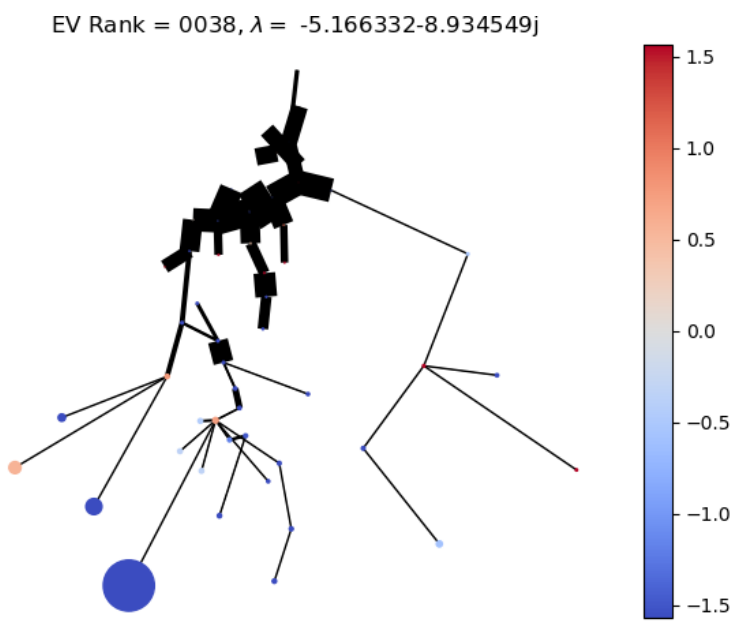

Fig. 9. Phase and magnitude of 38th ranked eigenvalue.

similar color and size will be coherent. Two observations are interesting: The complex phases are all very close to $\pm \pi / 2$. In the time response, this corresponds to a phase shift of $\pi$, so both clusters oscillate anti-phasic. Also, most nodes are in one, large cluster, with different magnitudes. Only a few nodes, included in a small, interconnected region are antiphasic. Those have smaller magnitude, but the magnitudes are not identical over all oscillators.

In contrast, the $38 \mathrm{~h}$ eigenvector components are displayed in figure 9. The magnitudes of a large number of nodes is almost zero, meaning that the node does not react to excitations of this mode. Some single nodes, especially larger blue circle, dominate this mode. Coherency is almost not found, except for the fact that many modes not oscillate at all.

The eigenvector properties can be investigated in a polar projection, as shown in figure 10. As seen earlier, most eigenvector components for the higher ranked eigenvalue are centered around zero, indicating no oscillation in this modes. 


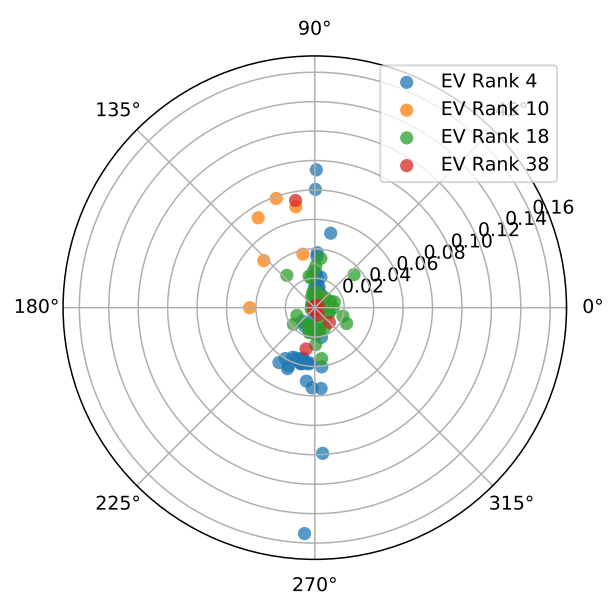

Fig. 10. Polar projection of eigenvector components for different modes.

Single nodes have a large, individual share of the dynamics in this mode. Fourth mode, the clustering of the most modes can be observed at a small magnitude around 260 degrees. The 18th eigenvector has most modes active at small magnitudes, centered around zero. The 38th mode has, as seen before, only a few active nodes, while most modes are almost zero. Also, noteworthy is the alignment of most eigenvalues around the $\pm \pi / 2$ axis $\left(90^{\circ}\right.$ to $\left.270^{\circ}\right)$. This corresponds to a low damping value in the system, as the undamped system is a hermitian generalized eigenvalue problem, where the eigenvectors will be purely imaginary.

\section{CONCLUSION}

In this work, the influence of the inertia and damping on the spectral properties of a linearized power system model was investigated. It was shown that the damping has, for physical relevant values, only small effect on the structure of the eigenvalues. The influence of the local parameters is mostly noted at the higher ranked eigenvalues, while the lower ranked eigenvalues are dominated by the global properties of the Laplacian matrix. Also, general questions of the robustness of the structure of the spectrum was investigated, with the result that the spectral properties are relatively robust for moderate changes in the relevant parameters.

A theoretical explanation of slow coherency is given in [15]. There, the case of $D=0$ was investigated analytically. It was shown that a network which can be partitioned in $q$ clusters, which are weakly connected to each other, will show slow coherency in the $q$ slowest modes. In the reduced realistic system as used in this work, the conditions for this theorem are not directly observed. As this condition is only sufficient, coherency can be observed in different systems as well.

In general, the structure of eigenvectors are a very difficult mathematical topic and only little analytical results are known to the authors. The most recent advance is proving an upper bound for the number of nodal domains (regions where the sign of the eigenvectors does not change) in homogeneous graphs [16]. The extension to the quadratic eigenvalue problem (and their complex nature) and to weighted, non-homogeneous graphs is not directly possible.

Even though the system does not respect the theoretical properties, the system shows coherent behavior, as shown in the last section. Future work will focus on the structure of the eigenvectors. While general theoretical results might be impossible to get, the idea is to investigate the undamped system (which simplifies the system from a QEP to a generalized eigenvalue problem) of a homogeneous complete graph with a general weight matrix $M$. The results will be perturbed by including weights in the complete graph, which should allow for insights of the structure of the eigenvectors of the undamped system.

\section{ACKNOWLEDGMENTS}

This project has received funding from the European union's Horizon 2020 research and innovation programme under Marie Sklodowska-Curie grant agreement No 675318

\section{REFERENCES}

[1] F. Dörfler and F. Bullo, "Synchronization in complex networks of phase oscillators: A survey," Automatica, vol. 50, no. 6, pp. 1539-1564, Jun. 2014.

[2] P. Ji and J. Kurths, "Basin stability of the Kuramoto-like model in small networks," The European Physical Journal Special Topics, vol. 223, no. 12, pp. 2483-2491, Oct. 2014.

[3] F. Dörfler and F. Bullo, "On the Critical Coupling for Kuramoto Oscillators," SIAM Journal on Applied Dynamical Systems, vol. 10, no. 3, pp. 1070-1099, 2011.

[4] M. Rohden, A. Sorge, D. Witthaut, and M. Timme, "Impact of network topology on synchrony of oscillatory power grids," Chaos: An Interdisciplinary Journal of Nonlinear Science, vol. 24, no. 1, p. 013123, Mar. 2014.

[5] A. E. Motter, S. A. Myers, M. Anghel, and T. Nishikawa, "Spontaneous synchrony in power-grid networks," Nature Physics, vol. 9, no. 3, pp. 191-197, Feb. 2013.

[6] Z. Assi Obaid, L. Cipcigan, and M. T. Muhssin, "Power system oscillations and control: Classifications and PSSs' design methods: A review," Renewable and Sustainable Energy Reviews, vol. 79, pp. 839849, Nov. 2017.

[7] F. Dorfler, M. Chertkov, and F. Bullo, "Synchronization in complex oscillator networks and smart grids," Proceedings of the National Academy of Sciences, vol. 110, no. 6, pp. 2005-2010, Feb. 2013.

[8] G. Filatrella, A. H. Nielsen, and N. F. Pedersen, "Analysis of a power grid using a Kuramoto-like model," The European Physical Journal B, vol. 61, no. 4, pp. 485-491, Feb. 2008.

[9] T. Nishikawa and A. E. Motter, "Comparative analysis of existing models for power-grid synchronization," New Journal of Physics, vol. 17, no. 1, p. 015012, Jan. 2015.

[10] F. Tisseur and K. Meerbergen, "The quadratic eigenvalue problem," SIAM review, vol. 43, no. 2, pp. 235-286, 2001.

[11] T. F. Walsh and D. M. Day, "Quadratic eigenvalue problems." Sandia National Laboratories, Tech. Rep., 2007.

[12] F. Dorfler and F. Bullo, "Kron Reduction of Graphs With Applications to Electrical Networks," IEEE Transactions on Circuits and Systems I: Regular Papers, vol. 60, no. 1, pp. 150-163, Jan. 2013.

[13] "Power Systems Test Case Archive - UWEE — http://labs.ece.uw.edu/," http://labs.ece.uw.edu/pstca/.

[14] F. Tisseur and N. J. Higham, "Structured Pseudospectra for Polynomial Eigenvalue Problems, with Applications," SIAM Journal on Matrix Analysis and Applications, vol. 23, no. 1, pp. 187-208, Jan. 2001.

[15] B. Ayazifar, "Graph spectra and modal dynamics of oscillatory networks," Ph.D. dissertation, Massachusetts Institute of Technology, 2002.

[16] J. C. Urschel, "Nodal decompositions of graphs," Linear Algebra and its Applications, vol. 539, pp. 60-71, Feb. 2018. 